\title{
The benchmark set for slopes generalization
}

\author{
Karel Stanek *, Petr Silhak, Aneta Ryglova \\ Dept. of Geography, Faculty of Science, Masaryk University, Brno, Czech Republic, karst@ geogr.muni.cz \\ * Corresponding author
}

Keywords: Automated cartographic generalization, slopes, benchmark

\begin{abstract}
:
We offer our initial task in automatizing the Czech Republic middle-scale topographic map work for map generalization benchmarking purposes. A terrain slope is defined as a place where the slope of the terrain is changing. The terrain slope is oriented, and there are small lines (hairs) on the right side of the slope (as figure 1 shows). We found that only $5 \%$ of slopes are not in the collision with another feature. After performing our generalization procedures, the largest group of conformal slopes with constraints consists of slopes parallelized or aligned with other line features (mainly roads, paths, rivers, or outlines of land-use boundaries).
\end{abstract}

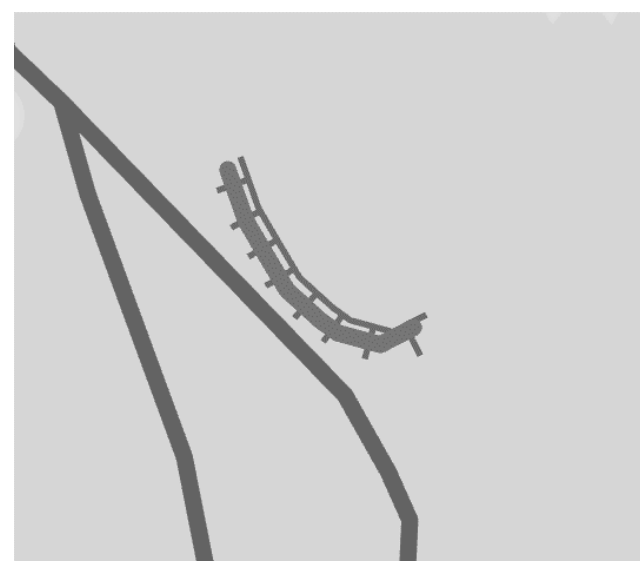

(a)

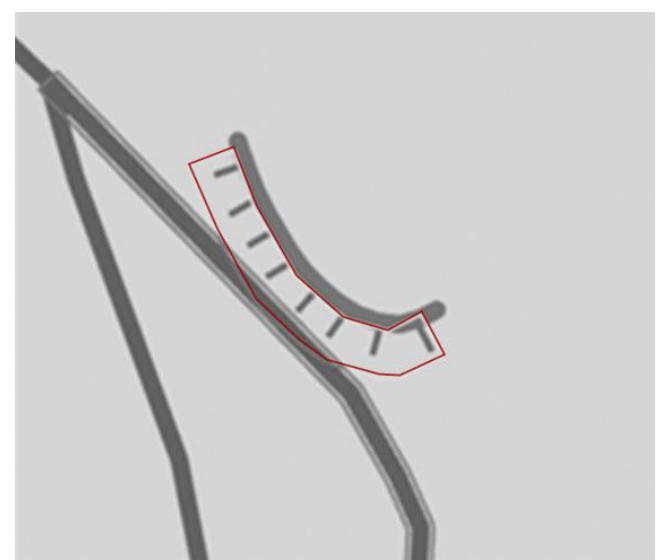

(b)

Figure 1. The collision between slope and path: (a) Slope will collide with path after using the proper symbology. The non-symbolized line near the slope is the initial slope; (b) Slope after automatic generalization. Radial displacement was chosen by software in this situation. The non-symbolized slope was almost completely identified with symbolized slope (position selected by a human operator). The light red outline is an asymmetric buffer of the original slope.

Results are from the manual map compilation (manually edited by human operators) of the CZ base topographic map 1:10 000 (ZTM10). In the case of the slopes processing, we sometimes cannot distinguish between constraints and rules (e.g., for certain combinations of linear elements, it is expected to align their shape, this constraint is very close to a rule because it includes the proposal of slope shape adjustment).

In this case, constraints mean slope parameters that create a collision of the slope with other features, including another slope. For example, the constraint can be a minimum distance between the symbolized slope and other symbolized features or the minimum slope length.

On the contrary, rules store information about solutions for the particular situation described by values of the constraints.

Basic demands on slopes are following:

1. The minimal distance of two unconnected objects on the ZTM10 map is $2 \mathrm{~m}$

2. In case of conflict, slopes are displaced (except with tree alleys)

3. Tree alleys can mask slopes

4. Metric parameters of the slopes in ZM10 are: the baseline is $0,13 \mathrm{~m}$, hairs are long $0,7 \mathrm{~m}$ and their distance is also $0,7 \mathrm{~m}$

5. The minimal length of the isolated slope on the ZTM10 is $50 \mathrm{~m}$ and $20 \mathrm{~m}$ in case of a close object 
6. If base of the slope line is closer than $2 \mathrm{~m}$ to another line feature, the slope is snapped to that line (in case of closeness to another slope base is base unified)

We use several shape modifications (simplification, smoothing, bend of the end part, bend of the intermediate part, trim, shapes harmonization, directional shrink, and radial displacement. There can be different approaches. The main goal is to get closer to the geometry after manual modification by human operators. However, in some cases, automatic processing can be much more consistent than humans.

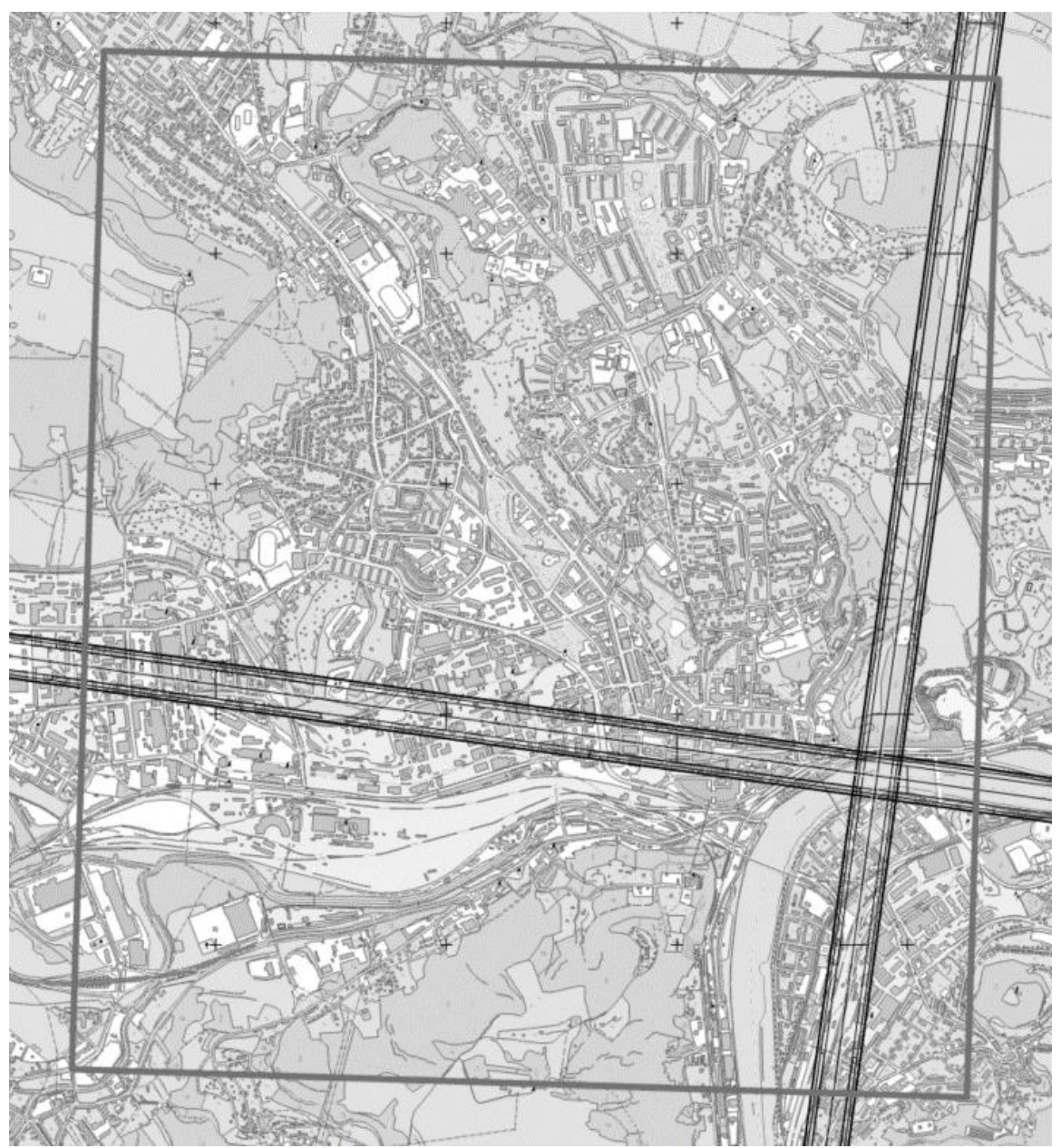

Figure 2. Dataset (DOI: 10.5281/zenodo.5745583) is represented by the ArcGIS package, geodatabase, and shapefiles which contains the original unmodified terrain slopes (SlopesUnmodified layer), terrain levels manually edited by mapping agency operators (SlopedModifiedByOperator layer), other data in the OtherRawData group (a group of layers that are in collision with the slopes most often), extent data (Extent layer) and symbolized data showing the final visualization (MapFrameDataSymbolized layer). The content of the data is described in the reading.me file.

Our benchmark dataset would allow the comparison of the following aspects:

1. Visualized result

2. Positional difference to the source - vertices distance statistics (average, median, sum)

3. Positional difference to human processed slopes

4. Shape difference to human processed slopes - identity, buffer overlap value, angularity differences

5. Processing description - count of operator and parameter, including combinations

6. Time of processing - with system and software information

Constraints and rules conformity (including rules and the topological consistency) are expected.

The data were provided by the Czech Mapping Agency (Č́UZK) within the project of automatic creation of Czech topographic maps of medium scales. 